\title{
ON THE CONSTRUCTION OF PERIODIC MAPS WITHOUT FIXED POINTS ${ }^{1}$
}

\author{
P. E. CONNER AND E. E. FLOYD
}

1. Introduction. We will consider the existence problem for fixed points of periodic maps. When the period is prime power, the theorem of P. A. Smith is available [5]. That is, if $X$ is a locally compact, finite dimensional space whose $\bmod p$ Čech cohomology groups are trivial, and if $T: X \rightarrow X$ is of period $p^{n}$ where $p$ is a prime, then $T$ has a fixed point. It is shown here that such propositions are false in case the period is not prime power.

In $\$ 4$ we show that if $r$ is a positive integer greater than one which is not prime power then there exists a compact, finite dimensional space $X$ with trivial integral Cech cohomology groups and a map $T: X \rightarrow X$ of period $r$ which is without fixed points. In $\S 6$, with $r$ as above, it is shown that there exists a contractible manifold $M$ and a map $T: M \rightarrow M$ of period $r$ and without fixed points. It seems reasonable to conjecture that the manifold $M$ constructed is actually a Euclidean space $E^{n}$. If this could be proved, the Smith conjecture that periodic maps on $E^{n}$ have a fixed point would be answered in the negative.

2. Commuting maps; the prime power case. Our constructions will be based on the study of maps $f: X \rightarrow X$ which commute with a given periodic map $T$. In this section we point out some of the facts in the prime power case.

We say that an endomorphism $h$ of the group $G$ is nilpotent if for each $x \in G$ we have $h^{n}(x)=0$ for $n$ sufficiently large. In the following, $H^{i}(X, K)$ denotes the Cech cohomology group with coefficients in $K$, and $H^{0}(X ; K)$ denotes the reduced 0 -dimensional group.

(2.1) Suppose that $T$ is a periodic map of prime power period $p^{n}$ on the compact, nonempty, finite dimensional space $X$. Suppose also that $f: X \rightarrow X$ is a map such that $f T=T f$ and that $f^{*}: H^{i}\left(X, Z_{p}\right)$ $\rightarrow H^{i}\left(X, Z_{p}\right)$ is nilpotent for $i=0,1, \ldots$. If $F$ denotes the fixed point set of $T$, then $F$ is nonempty and $(f \mid F)^{*}: H^{i}\left(F, Z_{p}\right) \rightarrow H^{i}\left(F, Z_{p}\right)$ is also nilpotent for $i=0,1, \cdots$.

Proof. Consider the inverse system

Received by the editors October 11, 1958.

1 This research was supported in part by the United States Air Force through the Air Force Office of Scientific Research of the Air Research and Development Command. under contract AF 49(638)-72 at the University of Virginia 


$$
X \stackrel{f}{\leftarrow} X \stackrel{f}{\leftarrow} \underset{f}{\leftarrow} \cdots .
$$

Let $Y$ denote the inverse limit space whose points are the sequences $\left(x_{i}: x_{i} \in X\right.$ and $\left.f x_{i+1}=x_{i}\right)$. Since $f^{*}$ is nilpotent, the direct limit of

$$
H^{i}\left(X, Z_{p}\right) \stackrel{f^{*}}{\rightarrow} H^{i}\left(X, Z_{p}\right) \stackrel{f^{*}}{\rightarrow} \cdots
$$

is trivial, so that $H^{i}\left(Y, Z_{p}\right)=0$ for $i=0,1, \cdots$ Also $\operatorname{dim} Y \leqq \operatorname{dim} X$, so that $Y$ is finite dimensional. There is the periodic map $T^{\prime}: Y \rightarrow Y$ which maps $\left(x_{i}\right)$ into $\left(T x_{i}\right)$. By the Smith theorems referred to in the introduction [5], the fixed point set $F^{\prime}$ of $T^{\prime}$ is nonempty and $H^{i}\left(F^{\prime}, Z_{p}\right)=0$ for $i=0,1, \cdots$. Since $F^{\prime}$ is nonempty, then $F$ is nonempty.

Also $F^{\prime}$ is the inverse limit space of

$$
F \stackrel{f \mid F}{\longleftarrow} \underset{f \mid F}{\longleftarrow}: \text {. }
$$

so that $H^{i}\left(F^{\prime}, Z_{p}\right)$ is the direct limit of

$$
H^{i}\left(F, Z_{p}\right) \stackrel{(f \mid F)^{*}}{\longrightarrow} H^{i}\left(F, Z_{p}\right) \stackrel{(f \mid F)^{*}}{\longrightarrow} \cdots
$$

Since $H^{i}\left(F^{\prime}, Z_{p}\right)=0$, then $(f \mid F)^{*}$ is nilpotent. The theorem follows.

We now show by example that $(f \mid F)^{*}$ is not necessarily nilpotent when coefficients are in the group $Z$ of integers. The example is closely related to the deeper examples of $\$ 3$.

(2.2) Consider the involution $T: S^{2} \rightarrow S^{2}$ which is reflection in a great circle, and whose fixed point set $F$ is therefore a circle. There exists a map $f: S^{2} \rightarrow S^{2}$ with $f T=T f$, with $f$ homotopic to a constant, and with $f \mid F: S^{1} \rightarrow S^{1}$ of degree 2 .

Proof. Consider $S^{2}$ as the join $S^{0} \circ S^{1}$ of a 0 -sphere $S^{0}$ and a circle $S^{1}$. Let $T^{\prime}: S^{0} \rightarrow S^{0}$ denote the involution interchanging the two points of $S^{0}$. Let $T: S^{2} \rightarrow S^{2}$ denote the join $T^{\prime}$ o $i d$ which maps the line segment from $x_{0} \in S^{0}$ to $x_{1} \in S^{1}$ linearly onto the segment from $T^{\prime} x_{0}$ to $x_{1}$. Let $f^{\prime}: S^{1} \rightarrow S^{1}$ denote an arbitrary map of degree 2 . We extend $f^{\prime}$ to a map $f: S^{2} \rightarrow S^{2}$ satisfying the assertions of our theorem. Define $f$ so as to map the first third of the segment from $x_{0} \in S^{0}$ to $x_{1} \in S^{1}$ linearly onto the segment from $x_{0}$ to $x_{1}$, the middle third linearly onto the segment from $x_{1} \in S^{1}$ to $T^{\prime} x_{0} \in S^{0}$, and the final third linearly onto the interval from $T^{\prime} x_{0} \in S^{0}$ to $f^{\prime} x_{1} \in S^{1}$. It may be seen that $f T=T f$ and that $f \mid F=f^{\prime}$, which is of degree 2. It remains to be seen that $f$ is of degree 0 . We leave this to the reader, since we do not use the example further. 
3. Commuting maps; the composite case. In this section we give examples to show that the results of the preceding section cannot be extended to maps whose periods are not prime power. These examples form the basis for the remainder of the paper.

(3.1) Suppose that $r$ is a positive integer greater than one which is not prime power. There exists a periodic map $T: S^{3} \rightarrow S^{3}$ of the 3-sphere $S^{3}$ which is of period $r$ and is without fixed points, and there exists a map $f: S^{3} \rightarrow S^{3}$ with $f T=T f$ and with $f$ homotopic to a constant.

PROOF. Suppose $r=p_{1} p_{2}$, where $p_{1}$ and $p_{2}$ are relatively prime and greater than one. Let $T_{i}: S^{1} \rightarrow S^{1}$ denote rotation through $2 \pi / p_{i}$, $i=1,2$. Consider $S^{3}$ as the join $S^{1} \circ S^{1}$, consisting of a union of intervals from $x_{1} \in S^{1}$ to $x_{2} \in S^{1}$, one joining each $x_{1}$ in the first copy of $S^{1}$ in $S^{1} \circ S^{1}$ to each $x_{2}$ in the second copy of $S^{1}$ in $S^{1} \circ S^{1}$. Let $T=T_{1} \circ T_{2}$; that is, if $x=(1-t) x_{1}+t x_{2}$ let $T x=(1-t) T_{1} x_{1}+t T_{2} x_{2}$. Then $T$ is of period $r$, and without fixed points. Of course, $T$ is a well-known periodic map of $S^{3}$.

We now construct $f: S^{3} \rightarrow S^{3}$. Since $p_{1}$ and $p_{2}$ are relatively prime, there exist integers $n_{1}$ and $n_{2}$ with $n_{1} p_{1}+n_{2} p_{2}=-1$. Consider the maps $f_{i}: S^{1} \rightarrow S^{1}$ given in complex coordinates by $f_{i}(z)=z^{m_{i}}$. Then $f_{i} T_{i}=T_{i} f_{i}$ if $m_{i}=1 \bmod p_{i}$. Set $m_{i}=n_{i} p_{i}+1, i=1,2$. Define $f: S^{3} \rightarrow S^{3}$ so as to map the first third of the segment from $x_{1}$ in the first copy $S_{1}^{1}$ of $S^{1}$ in $S^{1} \circ S^{1}$ to $x_{2}$ in the second copy $S_{2}^{1}$ of $S^{1}$ in $S^{1} \circ S^{1}$ linearly onto the segment from $f_{1} x_{1}$ to $x_{2}$, the middle third linearly onto the segment from $x_{2} \in S_{2}^{1}$ to $x_{1} \in S_{1}^{1}$, and the final third linearly onto the interval from $x_{1} \in S_{1}^{1}$ to $f_{2} x_{2}$. Explicitly

$$
f\left((1-t) x_{1}+t x_{2}\right)=\left\{\begin{array}{l}
(1-3 t) f_{1} x_{1}+3 t x_{2}, 0 \leqq t \leqq 1 / 3 \\
(3 t-1) x_{1}+(2-3 t) x_{2}, 1 / 3 \leqq t \leqq 2 / 3 \\
(3-3 t) x_{1}+(3 t-2) f_{2} x_{2}, 2 / 3 \leqq t \leqq 1
\end{array}\right.
$$

It can be seen that $f T=T f$. The remainder of the proof will be devoted to showing that $f: S^{3} \rightarrow S^{3}$ is of degree 0 .

If $a \in S^{1}$ is represented by $e^{2 \pi i \alpha}$, where $\alpha$ is an irrational number, then $f^{-1}(a / 2+a / 2)$ consists of three types of points

1. $5 x_{1} / 6+a / 6 ; f_{1}\left(x_{1}\right)=a, x_{1} \in S^{1}$;

2. $a / 2+a / 2$

3. $5 a / 6+x_{2} / 6 ; f_{2}\left(x_{2}\right)=a, x_{2} \in S^{1}$.

We observe that $f$ is differentiable near each point of $f^{-1}(a / 2+a / 2)$; it is also orientation preserving except at the point 2 . Hence ${ }^{2} \operatorname{deg} f$ $=\operatorname{deg} f_{1}-1+\operatorname{deg} f_{2}=n_{1} p_{1}+n_{2} p_{2}+1=0$. The theorem is proved.

2 We are indebted to the referee for suggesting the counting argument, which is shorter than our original proof. 
4. A fixed point free map on a compact acyclic space. A special case of the theorem of Smith [5] implies that if $T: X \rightarrow X$ is of prime power period where $X$ is a compact, finite dimensional space with $H^{i}(X, Z)=0$ for $i=0,1, \cdots$, then $T$ has a fixed point. We show that this holds only if the period is prime power.

(4.1) Suppose that $r$ is a positive integer greater than one which is not prime power. There exists a compact finite dimensional space $X$ with $H^{\imath}(X, Z)=0$ for $i=0,1, \cdots$ and a map $T: X \rightarrow X$ of period $r$ such that $T$ has no fixed points.

Proof. We use the technique of the proof of (2.1). Consider the map $T: S^{3} \rightarrow S^{3}$ of period $r$ and the map $f: S^{3} \rightarrow S^{3}$ constructed in (3.1). Then $T$ is without fixed points, $f T=T f$, and $f$ is homotopic to a constant. Consider the inverse mapping system

$$
S^{3} \stackrel{f}{\leftarrow} S^{3} \stackrel{f}{\leftarrow} S^{3} \stackrel{f}{\leftarrow} \ldots .
$$

The limit space $X$ of this system has $H^{i}(X, Z)=0$ for $i=0,1, \cdots$, since $f^{*}=0$. The map $T: S^{3} \rightarrow S^{3}$ generates a map $T^{\prime}: X \rightarrow X$ which maps $\left(x_{i}\right)$ into $\left(T x_{i}\right) . T^{\prime}$ is of period $r$ and without fixed points. The conclusion follows.

5. Fixed point free maps on contractible complexes. We construct in this section a star-finite, finite dimensional contractible complex $X$ together with a periodic simplicial map $T: X \rightarrow X$ which has no fixed points. The period $r$ of $T$ may be any positive integer greater than one which is not prime power. The fundamental device (other than (3.1)) used in the construction is that of an iterated mapping cylinder, a concept to which we have been led by study of an example of Eilenberg [1].

The mapping cylinders that we use are possibly slightly different from the usual, due to a desire to obtain polyhedra.

(5.1) Definition. Suppose $X$ and $Y$ are finite complexes, and that $f: X \rightarrow Y$ is simplicial. Suppose also that the vertices of $X$ are partially ordered by an ordering < under which the vertices of any simplex of $X$ are simply ordered. It will be convenient to suppose that $X$ and $Y$ are embedded as disjoint subcomplexes of a simplex $s$. The simplicial mapping cylinder $C$ of $f$ denotes the finite complex consisting of the union of $X$, of $Y$, and of all faces of simplices spanned by $x_{0}, \cdots, x_{k}, f x_{k}, \cdots, f x_{n}$ (repetitions are possible) where $x_{0}<\cdots$ $<x_{n}$ are vertices of a simplex of $X$ and where $0 \leqq k \leqq n$. Call $X$ the beginning of $C$ and $Y$ the end of $C$.

(5.2) Suppose $f: X \rightarrow Y$ as in (5.1). The simplicial mapping cylinder $C$ may be contracted (in $C$ ) into its end $Y$. If $f$ is homotopic to a con- 
stant, then the beginning $X$ of $C$ may be contracted to a point in $C$.

Proof. The set of vertices of $C$ is the union of the vertices of $X$ and the vertices of $Y$. Partially order the vertices of $C$ by taking the given partial ordering on $X$, by simply ordering the vertices of $Y$, and by defining each vertex of $X$ to be less than each vertex of $Y$ Triangulate $C \times I$. If $v_{0}<v_{1}<\cdots<v_{n}$ span a simplex of $C$ and $0 \leqq k \leqq h$, then

$$
v_{0} \times 0, \cdots, v_{k} \times 0, v_{k} \times 1, \cdots, v_{n} \times 1
$$

span a simplex of $C \times I$. We define now the simplicial contracting map $F$. Define $F(v \times 0)=v$ for all vertices $v$ of $C$. If $v$ is a vertex of $X$, let $F(v \times 1)=f v$; if $v$ is a vertex of $Y$, let $F(v \times 1)=v$. It may be checked that $F$ extends to a simplicial map $F: C \times I \rightarrow C$, and that $F$ contracts $C$ into $Y$.

If we consider $F \mid X \times I$ we obtain a homotopy of the inclusion $X \subset C$ to the composition $X \rightarrow^{f} Y \subset C$ in $C$. If $f$ is homotopic to a constant, then the beginning $X$ of $C$ may be contracted to a point in $C$.

(5.3) Definition. Suppose that $X$ is a finite polyhedron with triangulation $\Delta_{1}$, and that $f: X \rightarrow X$ maps an $n$th barycentric subdivision $\Delta_{2}$ of $\Delta_{1}$ simplicially into $\Delta_{1}$. Suppose $<$ is the natural partial ordering of a barycentric subdivision: $x_{0}<x_{1}$ if the simplex of which $x_{0}$ is barycenter is a face of the simplex of which $x_{1}$ is barycenter. In the simplicial mapping cylinder $C$, the beginning of $C$ occurs with triangulation $\Delta_{2}$ and the end of $C$ with triangulation $\Delta_{1}$. Let $C^{\prime}$ denote the $n$th barycentric subdivision of $C$ modulo the beginning of $C$. Then, in $C^{\prime}$, the beginning and end both occur with triangulation $\Delta_{2}$.

Let now $C_{1}, C_{2}, \cdots$ be a sequence of disjoint copies of $C^{\prime}$. Let $C_{\infty}$, the iterated mapping cylinder of $f$, denote the infinite complex obtained from $C_{1} \cup C_{2} \cup \ldots$ by identifying the end of $C_{i}$ and the beginning of $C_{i+1} . C_{\infty}$ is a star-finite, finite dimensional complex.

(5.4) If $f: X \rightarrow X$, as in (5.3), is homotopic to a constant, then $C_{\infty}$ is contractible.

Proof. Consider the subset $C_{1} \cup \ldots \cup C_{n}$ of $C_{\infty}$, with the end of $C_{i}$ identified with the beginning of $C_{i+1}$. Repeated use of (5.2) yields a contraction, in $C_{1} \cup \ldots \cup C_{n}$, of $C_{1} \cup \ldots \cup C_{n}$ into the end of $C_{n}$. Since $f$ is homotopic to a constant, the end of $C_{n}$ is contractible to a point in $C_{n+1}$. Hence $C_{1} \cup \ldots \cup C_{n}$ is contractible to a point in $C_{1} \cup \ldots \cup C_{n+1}$. All the homotopy groups of $C_{\infty}$ are therefore trivial, and $C_{\infty}$ is contractible.

(5.5) Theorem. Suppose that $r$ is a positive integer greater than one which is not prime power. There exists a star-finite, four dimensional 
contractible complex $X$ and a simplicial map $T: X \rightarrow X$ of period $r$ and without fixed points.

Proof. Consider the map $T: S^{3} \rightarrow S^{3}$ constructed in (3.1) as $T=T_{1} \circ T_{2}$. Now $T_{1}$ and $T_{2}$ are simplicial in certain triangulations $D_{1}$ and $D_{2}$ of $S^{1}$. There is a natural join triangulation $\Delta_{1}$ of $S^{1} \circ S^{1}$ under which $T$ is simplicial. It is no restriction to suppose that if $v$ is a vertex of $\Delta_{1}$ for which $v$ and $T^{i} v$ span an edge of $\Delta_{1}$, then $v=T^{i} v$.

Consider next the map $f: S^{3} \rightarrow S^{3}$ of (3.1) with $f T=T f$ and $f$ homotopic to a constant. There exists an $n$th barycentric subdivision $\Delta_{2}$ of $\Delta_{1}$ such that $f$ has a simplicial approximation from $\Delta_{2}$ to $\Delta_{1}$. That is, for each vertex $v$ of $\Delta_{2}$

$$
f\left(S t_{\Delta_{2}} v\right) \subset S t_{\triangle_{1}} w
$$

for some vertex $w$ of $\Delta_{1}$. Select one vertex $v$ from each $T$-orbit of vertices of $\Delta_{2}$. For such a $v$, select any vertex $w$ of $\Delta_{1}$, for which $\left(^{*}\right)$ holds and define $w=f^{\prime} v$. Operating on $\left({ }^{*}\right)$ with $T^{i}$ and using $f T^{i}=T^{i} f$,

$$
f\left(S t_{\Delta_{2}} T^{i} v\right) \subset S t_{\Delta_{1}} T^{i} f^{\prime}(v) .
$$

Define $f^{\prime}\left(T^{i} v\right)=T^{i} f^{\prime} v$. We must show that $f^{\prime}$ is uniquely defined. Suppose $T^{i} v=T^{i} v$. Then

$$
f\left(S t_{\Delta_{2}} T^{i} v\right) \subset S t_{\Delta_{1}} T^{i} f^{\prime}(v) \cap S t_{\Delta_{1}} T^{i} f^{\prime}(v) .
$$

Hence $T^{i} f^{\prime}(v)$ and $T^{i} f^{\prime}(v)$ span an edge of $\Delta_{1}$. Hence, by the requirement imposed on $\Delta_{1}, T^{i} f^{\prime}(v)=T^{j} f^{\prime}(v)$ and $f^{\prime}$ is uniquely defined.

Hence we have obtained a simplicial approximation $f^{\prime}: \Delta_{2} \rightarrow \Delta_{1}$ of $f$ with $f^{\prime} T=T f^{\prime}$. Also $f^{\prime}$ is homotopic to a constant, being homotopic to $f$. Let $X$ denote the iterated mapping cylinder $C_{\infty}$ of $f^{\prime}$. Now $T: S^{3} \rightarrow S^{3}$ is simplicial in both $\Delta_{1}$ and $\Delta_{2}$. It also preserves the order of (5.3) on vertices of $\Delta_{2}$. $T$ induces a periodic map on the simplicial mapping cylinder $C$ of $f^{\prime}: X \rightarrow X$ and also of the subdivision $C^{\prime}$ of $C$. Hence $T$ induces a map of period $r$ of $C_{\infty}$ which has no fixed points. The theorem follows.

6. Fixed point free maps on contractible manifolds. In this section we use a regular neighborhood technique used before $[3 ; 4]$ to expand the example of (5.5) into an example on a manifold. It is plausible that the manifold constructed is $E^{n}$; however, the J. H. C. Whitehead theory of regular neighborhoods [6] has been developed in full only for finite complexes so that we are not able to draw any immediate conclusion from it.

(6.1) Theorem. Suppose that $r$ is a positive integer greater than one 
which is not prime power. There exists a contractible manifold $M$ and a periodic map $S: M \rightarrow M$ of period $r$ which has no fixed points.

Proof. According to (5.5) there exists a star-finite, four dimensional contractible complex $K$ and a simplicial map $T: K \rightarrow K$ without fixed points. It is no restriction [3, p. 431] to suppose $K$ embedded as a subcomplex of triangulated Euclidean space $E^{n r}$ for some $n$, and to suppose that $T$ on $K$ is $S \mid K$ where $S$ is the cyclic permutation

$$
\left(x_{1}, \cdots, x_{r}\right) \rightarrow\left(x_{2}, \cdots, x_{r}, x_{1}\right), \quad x_{i} \in E^{n} .
$$

One may also suppose $S$ simplicial on all of $E^{n r}$. Consider the regular neighborhood $M$ of $K$; that is, the union of all open stars of $S d^{2} K$ relative to $S d^{2} E^{n r}$. Since $S(K) \subset K$ then $S(M) \subset M$. Now the fixed point set $F$ of $S$ is a subcomplex of $E^{n r}$, disjoint from $K$. Hence $F$ is disjoint from $M$. Therefore $S: M \rightarrow M$ is of period $r$ and without fixed points. Also $K$ is a deformation retract of $M[2$, p. 70]; since $K$ is contractible, so is $M$. The theorem follows.

\section{BIBLIOGRAPHY}

1. S. Eilenberg, On a theorem of $P$. A. Smith concerning fixed points for periodic transformations, Duke Math. J. vol. 6 (1940) pp. 428-437.

2. S. Eilenberg and N. Steenrod, Foundations of algebraic topology, Princeton, 1952.

3. E. E. Floyd, Fixed point sets of compact abelian Lie groups of transformations, Ann. of Math. vol. 66 (1957) pp. 30-35.

4. E. E. Floyd and R. W. Richardson, Jr., An action of a finite group on an n-cell without stationary points, Bull. Amer. Math. Soc. vol. 65 (1959) pp. 73-76.

5. P. A. Smith, Fixed point theorems for periodic maps, Amer. J. Math. vol. 63 (1941) pp. 1-8.

6. J. H. C. Whitehead, Simplicial spaces, nuclei and m-groups, Proc. London Math. Soc. vol. 45 (1939) pp. 243-327.

UNIVERSITY OF VIRGINIA 